\title{
De Novo Sequencing of Complex Mixtures of Heparan Sulfate Oligosaccharides
}

\author{
Rongrong Huang, Chengli Zong, Andre Venot, Yulun Chiu, Dandan Zhou, Geert-Jan Boons, \\ and Joshua S. Sharp* \\ Complex Carbohydrate Research Center, University of Georgia, Athens, Georgia 30602, United \\ States
}

\begin{abstract}
Here, we describe the first sequencing method of a complex mixture of heparan sulfate tetrasaccharides by LC-MS/MS. Heparin and heparan sulfate (HS) are linear polysaccharides that are modified in a complex manner by $\mathrm{N}$ - and $\mathrm{O}$-sulfation, $\mathrm{N}$-acetylation, and epimerization of the uronic acid. Heparin and HS are involved in various essential cellular communication processes. The structural analysis of these glycosaminoglycans is challenging due to the lability of their sulfate groups, the high heterogeneity of modifications, and the epimerization of the uronic acids. While advances in liquid chromatography (LC) and mass spectrometry (MS) have enabled compositional profiling of HS oligosaccharide mixtures, online separation and detailed structural analysis of isomeric and epimeric HS mixtures has not been achieved. Here, we report the development and evaluation of a chemical derivatization and tandem mass spectrometry method that can separate and identify isomeric and epimeric structures from complex mixtures. A series of well-defined synthetic HS tetrasaccharides varying in sulfation patterns and uronic acid epimerization were analyzed by chemical derivatization and LC-MS/MS. These synthetic compounds made it possible to establish relationships between HS structure, chromatographic behavior and MS/MS fragmentation characteristics. Using the analytical characteristics determined through the analysis of the synthetic HS tetrasaccharide standards, an HS tetrasacharide mixture derived from natural sources was successfully sequenced. This method represents the first sequencing of complex mixtures of HS oligosaccharides, an essential milestone in the analysis of structure-function relationships of these carbohydrates.
\end{abstract}

\section{Graphical Abstract}

\footnotetext{
"Corresponding Author: Tel.: (662) 915-1758. Fax: (662) 915-5638. jsharp@ olemiss.edu.

The authors declare no competing financial interest.

Supporting Information

The Supporting Information is available free of charge on the ACS Publications website at DOI: 10.1021/acs.anal-chem.6b00519.
}

MS/MS data of derivatized tetrasaccharides, a table of HS tetrasaccharide sequences identified from natural source, and detailed description of an example of MS/MS data interpretation for derivatized oligosaccharides (PDF). 


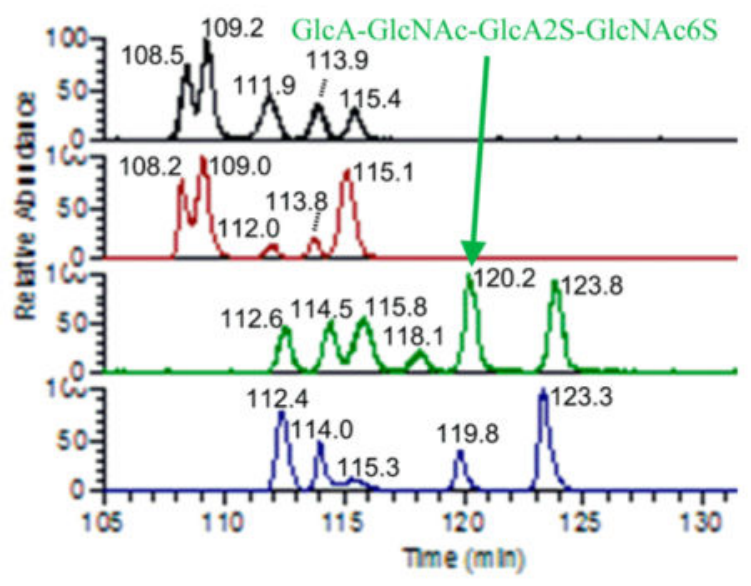

Glycosaminoglycans (GAG) are a family of negatively charged linear polysaccharides consisting of repeating disaccharide units, among which heparan sulfate (HS) is the most heterogeneous class with variability in O-sulfation positions, amine group modifications, and uronic acid epimerization. ${ }^{1,2} \mathrm{HS}$ is found on the surface of almost all mammalian cells and in the extracellular matrix, ${ }^{3,4}$ where it mediates a wide range of key biochemical and developmental processes, ${ }^{5-8}$ as well as pathological pathways. ${ }^{9-12}$ Heparin shares a basic structure with HS, differing in the extent of modification of the polysaccharide. Although a few function-specific heparin/HS motifs have been elucidated, such as the heparin pentasaccharide for binding to antithrombin III and inhibiting coagulation, ${ }^{13,14}$ there are numerous other heparin/HS sequences responsible for certain cellular functions that are still unknown. ${ }^{15,16}$ The need to better understand the structure/function relationships of HS has driven the development of advanced analytical methods for detailed structural characterization of these biomolecules. ${ }^{16-18}$ However, the heterogeneous nature of HS, caused by the postpolymerization modifications during biosynthesis, has made their structure determination a highly challenging task.

While advances in liquid chromatography (LC) and mass spectrometry (MS) make it possible to analyze many types of biomolecules in a sensitive and high throughput manner, serious challenges remain for structural sequencing of heparin/HS. ${ }^{19-21}$ This problem is mainly due to the chemical instability of sulfate groups and the high structural heterogeneity leading to considerable difficulties in separating isomeric structures. Efforts have been made to develop MS-based analytical methods for heparin/HS disaccharide analysis, oligosaccharide profiling, and tandem mass spectrometry (MS/MS)-based sequencing. ${ }^{22}$ Disaccharide analysis, a general and useful analytical method for the characterization of GAG populations, involves exhaustive depolymerization of intact heparin/HS into disaccharides followed by LC-MS for qualitative and quantitative analysis of the resulting disaccharides. ${ }^{23,24}$ While this method provides compositional information, it does not reveal the sequence information on oligosaccharide domains of the polysaccharide chains, which are considered to be the minimum sequences required by specific protein-HS binding interactions. Oligosaccharide profiling, on the other hand, can provide compositions and abundances of oligosaccharides with moderate lengths by performing partial depolymerization and LC-MS analysis. ${ }^{25,26}$ Oligosaccharides can be separated based on 
their size and degree of sulfation, and composition information (such as chain length, degree of sulfation, and number of acetyl groups) can be obtained by accurate mass measurement. Sequence information is not obtained from oligosaccharide profiling, and no information is obtained on uronic acid epimerization. While compositional profiling is useful for mixture characterization, the specific sequence of modifications is crucial to the specific biological functions of the heparin/HS oligosaccharide. ${ }^{15,18}$

Numerous difficulties exist for the MS/MS-based sequencing of heparin/HS oligosaccharides, among which sulfate loss during fragmentation is the major obstacle. Attempts have been reported recently to address this problem. Studies have shown that the sulfate loss during the collision induced dissociation (CID) can be minimized by lowering the degree of protonation through charge state manipulation and proton-sodium exchange. ${ }^{27}$ An alternative fragmentation method that has been used for MS/MS sequencing of heparin/HS is electron detachment dissociation or negative electron transfer dissociation, ${ }^{28-34}$ which under proper conditions seems to fragment heparin/HS with minimal loss of sulfates and can often differentiate between uronic acid epimers in pure oligosaccharide samples. Another potential solution is the replacement of the labile sulfates with stable acetyl groups by permethylation, desulfation and reacetylation before CID MS/MS analysis. ${ }^{35,36}$ We have demonstrated that this approach can successfully be employed for the sequencing of isomeric chondroitin sulfate (CS) oligosaccharides and synthetic HS oligosaccharides. A similar approach was reported by Lei et al. using permethylation, acidic solvolysis of sulfates, followed by trideuteropermethylation; however, this approach required harsher conditions for desulfation leading to peeling reactions in the. Also, the use of trideuteropermethylation prevents separation of the postlabeling oligosaccharides by chromatography due to the very similar chromatographic profiles of methyl and trideuteromethyl groups. ${ }^{37}$

Mass spectrometry is quite capable of separating nonisobaric heparin/HS oligosaccharides in the gas phase with very high resolution, making the technologies mentioned above useful for the sequencing of such analytes. However, in order to obtain unambiguous sequence identification by MS/MS for complex mixtures of heparin/HS oligosaccharides, such as those that would exist in the natural polymer, isomers need to be separated at least partially before the subsequent fragmentation. Several MS-compatible LC systems have been studied, including size-exclusion chromatography (SEC), ${ }^{38}$ hydrophilic interaction chromatography (HILIC), ${ }^{26,39}$ porous graphitized carbon (PGC), ${ }^{40,41}$ chromatography, and reversed phase ion pairing (RPIP). ${ }^{42,43}$ These techniques are effective for either disaccharide analysis or oligosaccharide profiling, however, the separation of isomeric complex mixtures of HS oligosaccharides has not been achieved by these methods. Ion mobility spectrometry (IMS), a group of technologies for gas-phase separation of ions, has also been reported recently for the separation of heparin/HS oligosaccharides. ${ }^{44,45}$ While this type of gas-phase separation can be employed for underivatized isomers and coupled with the use of appropriate MS/MS methods to sequence small oligosaccharides, the requirement of subtle instrumental tuning specific for each sample limits their application for robust and high throughput analysis. Additionally, there are still unresolved effects of gas-phase conformation and cation adduction state on the measured mobility. While future work shows promise in the 
application of IMS toward separation of isomers and epimers, they are currently not suited for analysis of complex mixtures of heparin/HS oligosaccharides.

As an alternative strategy, a chemical derivatization scheme was developed that employs the most widely used LC-MS/MS platform for separation and sequencing of heparin/HS oligosaccharides. This scheme involves the position-specific replacement of labile, hydrophilic groups of the heparin/HS oligosaccharide with stable hydrophobic groups, a modification and repurposing of a chemical derivatization scheme that was initially reported by Dell et al. for GAG analysis by FAB. ${ }^{46}$ While we previously demonstrated the ability of our method to perform de novo sequencing of heparin/HS oligosaccharides, the analysis of isomeric and epimeric HS oligosaccharides was not achieved due to the limited availability of isomeric HS oligosaccharide standards. ${ }^{35}$ Here, we report the development and validation of this technology for the separation and sequencing of isomeric HS tetrasaccharides. This further development of the technology was possible due to the creation of a library of synthetic HS tetrasaccharides varying in degree and position of sulfation and uronic acid epimerization. While this library is not exhaustive, it is sufficiently broad to allow the development of LC conditions, as well as the evaluation of fragmentation patterns specific for certain modification positions and epimerizations. The RPLC selectivity of isomers and epimers was systematically studied with the use of a fused core-porous shell C18 stationary phase, and structural information from the retention times of derivatized heparin/HS provides valuable structural information, especially regarding uronic acid epimerization states. The improved separation, coupled with the de novo sequencing using the derivatization-CID MS/MS method, made it possible for the first time to sequence a defined complex mixture of twenty-one synthetic HS tetrasaccharides, including many isomers and epimers. The utility of this method was demonstrated through the LC-MS/MS analysis of a mixture of HS tetrasaccharides obtained by enzymatic depolymerization of native HS, which represents the first report of LC-MS/MS to sequence a complex mixture of native HS tetrasaccharides.

\section{EXPERIMENTAL SECTION}

\section{Synthetic HS Tetrasaccharides Preparation}

HS tetrasaccharides were synthesized and purified as previously described ${ }^{47}$ The structures of the synthetic compounds were confirmed by NMR spectroscopy and mass spectrometry. Compounds were prepared with varying degrees and patterns of sulfation as well as uronic acid epimerization, including a series of tetrasaccharides with alkyl linker (Table 1) and two tetrasaccharides with free reducing end (GlcA-GlcNAc6S-GlcA-GlcNAc6S and GlcAGlcNAc6S-IdoA-GlcNAc6S).

\section{Native HS Tetrasaccharide Mixture Preparation}

An HS tetrasaccharide mixture was prepared by enzymatic digestion of intact HS (Celsus Inc., Cincinnati, OH), followed by gel filtration purification. Briefly, $100 \mathrm{mg} \mathrm{HS}$ and $0.05 \mathrm{IU}$ heparinase III (IBEX Technologies Inc., Quebec, Canada) were mixed in $1.5 \mathrm{~mL}$ of buffer of $50 \mathrm{mM}$ sodium acetate and $0.4 \mathrm{mM}$ calcium acetate. The solution was incubated at $37{ }^{\circ} \mathrm{C}$ for a total of $48 \mathrm{~h}$, with an additional aliquot of 0.05 IU heparinase III added after $24 \mathrm{~h}$ 
incubation. The enzyme digestion was stopped by heating the solution at $100{ }^{\circ} \mathrm{C}$ for $10 \mathrm{~min}$. The depolymerized samples were subjected to gel filtration chromatography on a Bio-Gel $\mathrm{P}-10$ column using $10 \%$ ethanol solution containing $1 \mathrm{M} \mathrm{NaCl}$ as a mobile phase to obtain tetrasaccharide fractions, which was further desalted through a Sephadex G-15 column and dried in vacuo.

\section{Chemical Derivatization of HS Tetrasaccharides}

HS tetrasaccharides, except those with an alkyl linker at the reducing end, which cannot be reduced, were first reduced by sodium borohydride, as previously described, ${ }^{48}$ followed by desalting and lyophilization. This reduction is necessary to both eliminate epimerization at the reducing end, as well as prevent peeling of 3-O sulfated glucosamine during the permethylation step under basic conditions. ${ }^{49}$ Complete permethylation, desulfation and pertrideuteroacetylation were performed to replace the original sulfate groups with trideuteroacetyl groups. Detailed procedures have been described in our previous work for structural analysis of synthetic HS oligosaccharides. ${ }^{35}$ Briefly, the dried triethylammonium salts of HS tetrasaccharides (10-100 $\mu \mathrm{g})$ were permethylated according to Ciucanu methylation method ${ }^{50}$ using sodium hydroxide and methyl iodide in dimethyl sulfoxide (DMSO), followed by desalting using a C18 Sep-Pak cartridge (Waters Co.). The pyridinium salts of the permethylated products were formed by ion exchange over Dowex 50WX8 resin, then resuspended in DMSO containing 10\% methanol and incubated for $4 \mathrm{~h}$ at $95{ }^{\circ} \mathrm{C}$ to remove the sulfate groups. The dried desulfated products were then trideuteroacetylated by incubating with $25 \mu \mathrm{L}$ of $\mathrm{D}_{6}$-acetic anhydride in $175 \mu \mathrm{L}$ of pyridine at $50{ }^{\circ} \mathrm{C}$ overnight and solvent was dried under vacuum. The final derivatized products were resuspended in $10 \%$ acetonitrile/water at the concentrations specified below for subsequent LC-MS/MS analysis. Yield for this derivatization scheme was previously reported for dp4 at approximately $29.5 \%,{ }^{35}$ which is consistent with the data reported here. Sources of sample loss include both loss during sample workup and uronic acid $\beta$-elimination products during permethylation, observable as derivatized disaccharides.

\section{LC-MS/MS Analysis}

For structural analysis of each synthetic tetrasaccharide, an RPLC-MS/MS method was used. Buffer B was prepared as acetonitrile with $0.1 \%$ formic acid, and buffer A was $0.1 \%$ formic acid in water with or without $1 \mathrm{mM}$ sodium formate. HPLC was performed on Agilent LC 1100 series using a Halo C18 column with fused core-porous shell packing materials $(0.2 \times 50 \mathrm{~mm}, 2.7 \mu \mathrm{m}, 160 \AA$, Advanced Material Technology, Wilmington, DE). A linear gradient of 10-60\% buffer B over15 min was used, with a flow rate of $9 \mu \mathrm{L} / \mathrm{min}$ and a $2 \mu \mathrm{L}$ injection at a sample concentration of $0.1 \mu \mathrm{g} / \mu \mathrm{L}$. Mass spectrometry was performed on a Thermo LTQ-FT instrument with a hand-pulled fused silica needle electrospray emitter. Electrical contact for applying the electrospray voltage was made by a platinum wire lead contact to the LC eluant made at the LC-emitter junction via a microtee. Full MS in FT mode and CID-MS/MS spectra in ion trap mode were acquired in positive ion mode. External calibration of the instrument produced mass accuracy of $<5 \mathrm{ppm}$ for full MS spectra, which enabled the use of accurate mass measurement for composition determination of precursor ion. A data dependent MS/MS method was used, with the top 4 abundant precursor ions selected, to trigger CID-MS/MS fragmentation. Instrument parameters were 
set as spray voltage at $1-2 \mathrm{kV}$, capillary voltage at $40 \mathrm{~V}$, tube lens at $80 \mathrm{~V}$ and capillary temperature at $250{ }^{\circ} \mathrm{C}$. The collision energy for CID fragmentation was set at $40 \mathrm{~V}$.

For isomeric and/or epimeric HS tetrasaccharide mixture analyses, RPLC separations were performed on a longer column with similar $\mathrm{C} 18$ packing material $(0.2 \times 500 \mathrm{~mm}, 5.0 \mu \mathrm{m}$, $160 \AA$, Advanced Material Technology, Wilmington, DE). For separation of synthetic HS tetrasaccharides, a 140 min gradient was used from 35\% to 55\% buffer B. The gradient was started at $35 \%$ buffer B to allow for separation of any derivatized disaccharide products that may have formed from beta-elimination of the tetrasaccharides; such side-products elute between $\sim 35 \%$ and $\sim 40 \%$ buffer B. Repeatability of elution times of tetrasaccharide standards using this gradient ranged from $\sigma=0.28 \mathrm{~min}$ to $\sigma=2.1 \mathrm{~min}$ in a duplicate sample (data not shown). For separation of native HS tetrasacharide mixtures, an isocratic elution was also used at $44 \%$ buffer B in order to obtain better separation. A flow rate of $2 \mu \mathrm{L} / \mathrm{min}$ and a $2 \mu \mathrm{L}$ injection at a sample concentration of $1 \mu \mathrm{g} / \mu \mathrm{L}$ were used for both mixture analyses. Mass spectrometry methods were the same as described above. All mass spectra were annotated manually using the nomenclature proposed by Domon and Costello. ${ }^{51}$

\section{RESULTS AND DISCUSSION}

The derivatization protocol used for the modification of the heparin/HS tetrasaccharides is outlined in Figure 1. Briefly, the tetrasaccharides are reduced at the reducing end to eliminate anomerization, and then quantitatively permethylated. The permethylation protects all nonsulfated groups, as well as aiding in the assignment of sulfation sites for the GlcN sugars. This step is followed by a gentle chemical desulfation. Finally, the former sites of sulfation are chemically labeled by a trideuteroacetylation step. The derivatized tetrasaccharides were sufficiently hydrophobic for retention on a standard C18 RPLC column. For the HS tetrasaccharides with an alkyl linker, a quaternary amine was formed during the permethylation, leaving a permanent fixed positive charge at the reducing-end alkylamine chain (Figure 1).

\section{MS/MS Analysis of Synthetic HS Tetrasaccharids with Alkyl Linker}

In our previous work, we have demonstrated that chemical derivatization allowed successful sequencing of HS oligosaccharides by differentiating all possible modification patterns without requiring cross-ring cleavages, which are difficult to reliably obtain in high abundance. ${ }^{35}$ Here, MS/MS spectra were taken for each synthetic HS tetrasaccharide listed in Table 1. The introduction of the fixed charge on the reducing end linker generated clean, abundant product ion spectra that were comparable to previous results, where ionization was driven by protonation or sodiation of the derivatized heparin/HS oligosaccharide.

After derivatization, sites of $\mathrm{N}$-sulfation (which has been labeled during derivatization with a trideuteroacetyl group) can be differentiated from sites of native $\mathrm{N}$-acetylation by a mass shift of 3 Da. For example, compounds 2a-e have a 6 Da mass increase compared to compounds 1a-e (Table 1).

Compounds 2a-e all have two N-sulfates, while similar compounds 1a-e all have two Nacetyls. The same results were measured for compounds $\mathbf{4 a} \mathbf{a}-\mathbf{e}$ compared to $\mathbf{3 a}-\mathbf{f}$ for identical 
reasons. We were able to accomplish de novo sequencing of each synthetic tetrasaccharide based solely on glycosidic bond cleavage of the derivatized product. In order to illustrate the differentiation of positional modification isomers and uronic acid epimers, the interpretation of the MS/MS spectra for compounds 3a-f are detailed below, and the MS/MS spectra of these compounds are presented in Figure 2. MS/MS spectra for compounds 1a-e, 2a-e, and 4a-e are also provided in Supporting Information, Figure S-1A-C.

Initially, the composition (number of disaccharide repeats, number of $\mathrm{N}$-acetylations, free amines, $\mathrm{N}$-sulfations, 3-O-sulfations, and other $\mathrm{O}$-sulfations) is determined based on the mass measurement of the intact derivatized glycan. For derivatized HS tetrasaccharides of $\mathrm{m} / z$ 1148.609, the composition is HexA-GlcNAc-HexA-GlcNAc-linker with two O-sulfation sites, neither of which occur at the 3-O position of the GlcNAc. ${ }^{35}$

The next step was to determine the sites of each modification. The sites of O-sulfation have a mass increase of $31 \mathrm{Da}$ after derivatization compared to the nonsulfated sugar, indicating a site of trideuteroacetylation (+45 Da) in place of a methylation (+14 Da). For example, the $\mathrm{Y}_{3}$ ion (a fragment containing the three monosaccharides at the reducing end of the glycan, cleaved at the reducing side of the glycosidic oxygen) of compound 3a has an $\mathrm{m} / \mathrm{z}$ of 885 , indicating that the $\mathrm{Y}_{3}$ ion has a single $\mathrm{O}$-sulfation site. Since the parent ion has two Osulfation sites, the nonreducing end uronic acid is $\mathrm{O}$-sulfated at the only possible site for uronic acid O-sulfation, the 2-O position. The $\mathrm{Y}_{3}$ ions for compounds $\mathbf{3 b}-\mathbf{e}$ were all $31 \mathrm{Da}$ heavier $\left(\mathrm{m} / z\right.$ 916), indicating that the $\mathrm{Y}_{3}$ ion of these tetrasaccharides all contain two Osulfates, and therefore there is a nonsulfated uronic acid at the nonreducing end. The $\mathrm{m} / z$ of the $\mathrm{Y}_{2}$ ion is similarly diagnostic. Compound 3a has a $\mathrm{Y}_{2}$ ion of $\mathrm{m} / z 609$, indicating no sulfates on the reducing end disaccharide and placing the remaining O-sulfation on the internal GlcNAc at the 6-O position. Compounds $\mathbf{3 b}, \mathbf{3 d}$, and $\mathbf{3 f}$ have $\mathrm{Y}_{2}$ ions of $\mathrm{m} / z 640,31$ Da heavier than compound 3a, clearly indicating that these compounds have one O-sulfate on the reducing end disaccharide and one O-sulfate on the internal GlcNAc. Compounds $\mathbf{3 c}$ and $3 \mathbf{e}$ have $\mathrm{Y}_{2}$ ions of $\mathrm{m} / z$ 671, indicating the presence of two O-sulfates on the reducing end disaccharide and a nonsulfated internal GlcNAc. By following the glycosidic bond fragments ( $\mathrm{Y}$ and $\mathrm{Z}$ ions containing the reducing end, $\mathrm{B}$ and $\mathrm{C}$ ions containing the nonreducing end) and measuring the composition of each fragment, we could identify the backbone structure for compound 3a as HexA2S-GlcNAc6S-HexA-GlcNAc-linker, compounds $\mathbf{3 c}$ and $\mathbf{3 e}$ as HexA-GlcNAc-HexA2S-GlcNAc6S-linker, and compounds $\mathbf{3 b}, \mathbf{3 d}$, and $\mathbf{3 f}$ as HexA-GlcNAc6S-HexA-GlcNAc6S-linker. In every case, the sequence determined by de novo analysis of the MS/MS spectrum agreed with the structure of the synthetic compound confirmed by NMR.

While sulfation and acetylation positional isomers can be readily differentiated by the masses of fragment ions, epimers can be differentiated by the relative intensities of fragment ions, as shown in Figure 2. For pair of nonreducing end uronic acid epimers $\mathbf{3 b}$ and $\mathbf{3 f}$, the ratio of the relative intensity of $\mathrm{Y}_{3} / \mathrm{Y}_{2}$ ion may be used to differentiate the nonreducing terminal GlcA (3f) with $\mathrm{Y}_{3} / \mathrm{Y}_{2}$ ratio of 0.7 and terminal IdoA (3b) with $\mathrm{Y}_{3} / \mathrm{Y}_{2}$ ratio of 3.3, indicating that the nonreducing end iduronic acid significantly promotes cleavage of the glycosidic bond to its nonreducing end compared to the glucuronic acid. The same cleavage preference was also observed with the other four compounds in Group 3, where compound 
3a with IdoA2S at the nonreducing end had $\mathrm{Y}_{3} / \mathrm{Y}_{2}$ ratio of 2.0, but compounds $\mathbf{3 c}, \mathbf{3 d}$, and 3e with a nonreducing GlcA had a $\mathrm{Y}_{3} / \mathrm{Y}_{2}$ ratio of $0.2,0.7$, and 0.1 , respectively.

For the internal epimeric pairs 3c:3e and 3d:3f, we observed abundant $Z_{2}$ ions (fragments containing the reducing end of the sugar where the cleavage occurred to the nonreducing side of the middle glycosidic oxygen) for $\mathbf{3 e}$ and $\mathbf{3 f}$ that contain an internal GlcA residue, but not for compounds $\mathbf{3 c}$ and $\mathbf{3 d}$ that contain internal IdoA. In addition, $\mathrm{Z}_{2}$ ions were also observed for the other two compounds $\mathbf{3 a}$ and $\mathbf{3 b}$ that contain internal GlcA residue. This indicated that abundant $\mathrm{Z}_{2}$ ions may be used as diagnostic ion for differentiation of internal GlcA and IdoA residue, regardless of the sulfation of the uronic acid.

These trends hold true for the Groups 1, 2, and 4 tetrasaccharide epimeric pairs (Figure S1, Supporting Information). All compounds with nonreducing terminal GlcA/GlcA2S had $\mathrm{Y}_{3} / \mathrm{Y}_{2}$ ratio $<0.8$ and those with nonreducing terminal IdoA/IdoA2S had $\mathrm{Y}_{3} / \mathrm{Y}_{2}$ ratio $>1.5$. Abundant $Z_{2}$ ions were observed for all compounds with internal GlcA/GlcA2S, but not for those with internal IdoA/IdoA2S. These results indicate that MS/MS identification of epimers through the derivatization process is possible.

\section{LC Separation of Synthetic HS Tetramers with Alkyl Linker}

While MS/MS could differentiate sulfate positions and epimerizations for the pure synthetic tetrasaccharides, the analysis of complex mixtures requires the separation of the isomers prior to MS/MS analysis. For the separation of synthetic HS tetrasaccharides with alkyl linker, an approximately equimolar mixture of 21 synthetic tetrasaccharides was subjected to the chemical derivatization process, which was analyzed by the automated LC-MS/MS approach described in the Experimental Section. The reversed phase LC separation was performed on a $500 \mathrm{~mm} \mathrm{C} 18$ column packed with the HALO-5 fused core-porous shell particles, using a gradient of $30-50 \% \mathrm{~B}$ for $140 \mathrm{~min}$ and a flow rate of $2 \mu \mathrm{L} / \mathrm{min}$. As the extracted ion chromatogram (EIC) shows in Figure 3, baseline separation was achieved for most of the species in each groups of isomers, with average peak width of approximately 1 min. For the compounds derived from the $\mathrm{N}$-acetylated tetrasaccharides vs $\mathrm{N}$-sulfated tetrasaccharides, (e.g., compounds 1a-e vs compounds 2a-e) the only difference is trideutero versus trihydroacetylation of the amines. As shown in Figure 3, the isotopic labeling did not alternate the chromatography selectivity, leading to the coelution of compounds 1a-e and compounds 2a-e. However, the $6 \mathrm{Da}$ mass shift for the parent ion $\mathrm{M}^{+}$ allowed for clean separation of these analytes by mass spectrometry, making the MS/MS analysis able to clearly differentiate between the two groups of sequences.

We observed patterns in LC selectivity that could be correlated to the original degree of sulfation, sulfation positions and uronic acid epimerization. In general, tetrasaccharides with a higher degree of sulfation elute later. This observation is consistent with what we observed for longer HS oligosaccharides. ${ }^{35}$ Comparison of the sulfation positions and retention time for isomer pairs 3a:3b, 3c:3d, and 3e:3f reveals that tetrasaccharides with 2-O-sulfation on GlcA/IdoA eluted earlier than those modified by 6-O sulfation on GlcNAc/GlcNS, with retention time differences ranging from 1 to $4 \mathrm{~min}$. For epimer pairs, more clear patterns could be observed for their retention times: tetrasaccharides with all GlcA eluted last, tetrasaccharides with nonreducing terminal IdoA elute first, and tetrasaccharides with an 
internal IdoA elute in between the two. For example, compound 3d eluted 3.6 min later than compound $\mathbf{3 b}$ and 5.7 min earlier than compound $\mathbf{3 f}$ (Figure 3). Overall, each structural characteristic contributed to the chromatographic behavior of these synthetic HS tetrasaccharides, where sulfation degrees and uronic acid epimerizations seemed to have a larger effect than sulfation positions. The addition of LC selectivity information to MS/MS fragmentation data increases the confidence with which the structure of heparin/HS oligosaccharides can be assigned.

\section{Structural Analysis and LC Separation of Two Epimeric Synthetic HS Tetrasaccharides with Free Reducing End}

Two synthetic epimers with free reducing ends (GlcA-GlcNAc6S-GlcA-GlcNAc6S and GlcA-GlcNAc6S-IdoA-GlcNAc6S), which have the same saccharide structure as synthetic tetrasaccharides $\mathbf{3 f}$ and $\mathbf{3 d}$, were also subjected to chemical derivatization and LC-MS/MS analysis to determine the effect of the alkyl linker on LC selectivity and resolution. The LC chromatogram and MS/MS spectra were presented in Figure 4. Compared to compounds $3 \mathbf{d}$ and $\mathbf{3 f}$ (Figure 3), tetrasaccharides without linker eluted about $20 \mathrm{~min}$ later under the same chromatographic conditions (Figure 4A). The fixed positive charge on the alkyl linker after derivatization made compounds $\mathbf{3 d}$ and $\mathbf{3 f}$ relatively more hydrophilic than the two tetrasaccharides without linker, leading to an earlier elution time for compounds $\mathbf{3 d}$ and $\mathbf{3 f}$. In addition, the selectivity within the epimer pair was also different. While compound $\mathbf{3 d}$ with an internal IdoA and an alkyl amine linker eluted about 6 min earlier than compound $\mathbf{3 f}$ with an internal GlcA, GlcA-GlcNAc6S-IdoA-GlcNAc6S eluted about 3 min later than GlcA-GlcNAc6S-GlcA-GlcNAc6S when the alkyl linker was absent from the reducing end. Although the mechanisms causing such differences are unclear, baseline separation was still achieved, demonstrating the capability of our methodology for separation of native epimeric HS tetrasaccharides.

MS/MS spectra were also compared for GlcA-GlcNAc6S-IdoA-GlcNAc6S and GlcAGlcNAc6S-GlcA-GlcNAc6S in order to differentiate the epimerization (Figure 4B,C). While fragmentation of the protonated precursor ion yielded useful sequencing ions, the MS/MS spectra did not differentiate epimers (data not shown). Selecting the sodiated parent ion [M $+\mathrm{Na}]^{+}$, similar patterns were observed as for the differentiation between compound $\mathbf{3 d}$ and compound $3 \mathbf{f}$ (Figure 2). Abundant $Z_{2}$ ions were observed for GlcA-GlcNAc6S-GlcAGlcNAc6S containing an internal GlcA residue, but not for GlcA-GlcNAc6S-IdoAGlcNAc6S. In addition, the intensity ratio of $\mathrm{Y}_{3} / \mathrm{Y}_{2}<0.8$ was observed for both MS/MS spectra, which was consistent with what we observed for tetrasaccharides with alkyl linker containing a nonreducing terminal GlcA residue. These data suggest that the fragmentation characteristics of sodiated ions of derivatized heparin/HS oligosaccharides with free reduced reducing ends are comparable to those containing the alkyl quaternary amine linker, broadening the applicability of the sequencing method.

\section{LC-MS/MS Analysis of Native HS Tetrasaccharide Mixture}

To evaluate and apply our method for structural analysis of native HS oligosaccharide mixtures, an enzymatic digested HS tetrasaccharide mixture was prepared as described in Experimental Section. Unlike the synthetic tetrasaccharides, lyase digestion generates 
oligosaccharides with an unsaturated uronic acid on the nonreducing end, eliminating the uronic acid epimerization in the process. Prior to chemical derivatization and LC-MS/MS analysis, an MS spectrum was taken in negative ion mode to obtain compositional information (Figure S-2A). For each observed molecular weight (MW), all possible sequences (ignoring epimerizations) are listed together with the MW for the corresponding derivatization product (Figure S-2D). After sequential chemical derivatizations, LC-MS/MS analysis was performed and $\mathrm{m} / \mathrm{z}$ corresponding to each derivatized MW listed in Figure S-2D was extracted from the full MS scan. Three major compositions were observed: tetrasaccharides with two $\mathrm{N}$-acetyl groups and no O-sulfate group (MW of 956.458); tetrasaccharides with one $\mathrm{N}$-acetyl group, one $\mathrm{N}$-sulfate group, and no O-sulfate group (MW of 959.477), and tetrasaccharides with one $\mathrm{N}$-acetyl group, one $\mathrm{N}$-sulfate group, and one $\mathrm{O}$ sulfate group (MW of 990.490; Figure S-2B). Tetrasaccharides with no sulfation, one acetylation and one free amine were observed at derivatized MW 943.486, but at very low abundance; no MS/MS analysis was possible of this composition.

The same LC conditions as described in the previous section were used for the initial analysis, with all three species eluting between the synthetic tetrasaccharides with alkyl linker and the two without linker (data not shown). This range of retention times was anticipated, as these native tetrasaccharides had free reducing ends instead of alkyl linkers and all three observed species had fewer O-sulfation sites than the two synthetic tetrasaccharides with free reducing ends. In order to obtain further separation to allow clear interpretation of isomers, isocratic elution was used at 44\% B (Figure S-2C). The same three compositions were detected, with resolved peaks for MW 990.490. A total of nine structures were identified from the three major compositions observed for the enzyme-digested HS tetrasaccharide mixture, with a tenth structure possible as a minor component (see Supporting Information, Table S-1).

The derivatized MW of 956.458 for the first composition indicated a tetrasaccharide with two GlcNAc and no sulfation. MS/MS analysis of this ion validated this composition and a $\mathrm{Z}_{2} / \mathrm{Y}_{2}$ ion ratio of $\sim 0.9$ was measured for the sodiated precursor, indicating an internal GlcA and yielding a sequence of dHexA-GlcNAc-GlcA-GlcNAc-ol (Figure S-3A). The derivatized tetrasaccharide of MW 960.485 contains one GlcNAc and one GlcNS, with no O-sulfations. MS/MS analysis of the tetrasaccharide revealed that the most abundant $\mathrm{Y}_{1}$ and $\mathrm{Y}_{2}$ fragment ions were consistent with a GlcNAc at the reducing end, but minor $\mathrm{Y}_{1}$ and $\mathrm{Y}_{2}$ fragment ions consistent with a GlcNS at the reducing end could also be detected $3 \mathrm{Da}$ heavier than the fragments indicating the GlcNAc, both at approximately $20 \%$ the abundance of the GlcNS product ions (Figure S-3B). The $\mathrm{Z}_{2} / \mathrm{Y}_{2}$ product ion ratio for the sodiated precursor was approximately 1.0 , and as only one chromatographic peak was observed for this MW, we can conclude that both structures have an internal GlcA. These results indicate that both dHexA-GlcNS-GlcA-GlcNAc-ol and dHexA-GlcNAc-GlcAGlcNS-ol are present in the mixture, with the prior structure probably being the more abundant.

For the species with a derivatized MW of 990.490, three major LC peaks were observed coming from a total of eight theoretical possible sequences containing one GlcNAc, one GlcNS, and one O-sulfation not at the 3-O position, ignoring internal HexA epimerizations 
(Figure S-2D). The TIC of MS/MS scans of parent ion $[\mathrm{M}+2 \mathrm{H}]^{2+}(\mathrm{m} / \mathrm{z} 496.252)$ and a series of EICs of diagnostic $\mathrm{Y}$ ions were presented in Figure 5 to illustrate the method for sequencing of sulfation positions. A detailed description of the process used to assign the structures for this set of isomers is given in Supporting Information. Briefly, the elution profile of various diagnostic $\mathrm{Y}$ ions from the $[\mathrm{M}+2 \mathrm{H}]^{2+}$ charge state precursor allows for the identification of isomeric sequences that are only partially resolved by LC. If the EIC of a series of sequencing ions perfectly align by LC elution profile, then the sequencing ions can be assigned to a single analyte. However, if they do not align, the sequencing ions are assigned to separate analytes. This allows for the identification of sequences that are only partially resolved by LC, and was previously used in the $\mathrm{MS}^{\mathrm{n}}$ analysis of native CS oligosaccharides. ${ }^{36}$ The near-perfect EIC coelution of two mutually exclusive fragment ions can also be used to differentiate between positions of $\mathrm{N}$-sulfation and $\mathrm{N}$-acetylation, which differ after derivatization by only the position of three deuterons that is difficult to separate to any extend by RPLC. Epimerization is determined based on a combination of elution time (Figures 5 and S2-C) and the $\mathrm{Z}_{2}-\mathrm{Y}_{2}$ fragment ion ratio from the $[\mathrm{M}+\mathrm{Na}]^{1+}$ precursor ion (Figure S-4). Based on the biosynthetic pathway of HS, IdoA/IdoA2S residues only occur when a GlcNS residue is linked at the nonreducing side, ${ }^{52}$ which can be used to support assignments of IdoA. Using this basic strategy and the data in Figure 5 and Figures S-3 and S-4, nine structures were identified in the HS tetrasaccharide mixture, with a tenth possible structure present (Table S-1).

The EICs of specific product ions shown in Figure 5 represent a low intensity group of isomeric precursors, some of which show significant noise in the signal intensity. As shown in Figure S-2, the set of isomers of $\mathrm{m} / z 990.490$ (which Figure 5 focuses on) have the most number of components for what is the precursor ion with the lowest intensity. At the Y1 ion, we have high diversity (three structures present) and a low intensity product ion (the $\mathrm{Y} 1$ ion in Figure S-3 is representative of usual Y1 ion intensities for these samples); therefore, the signal-to-noise ratio of the measurement is relatively low making the product ion EIC noisy. For the $\mathrm{Y} 3$ ion, which is a relatively intense product ion with only two structures present in the mixture, the signal-to-noise ratio is much higher, with the resulting product ion EICs appearing less noisy. The $\mathrm{Y} 2$ ion is intermediate; it is an abundant product ion with high diversity, resulting in a moderate signal-to-noise ratio. The noisiness of each product ion EICs is wholly a function of signal intensity.

\section{CONCLUSIONS}

The research described here represents the first successful separation and structural sequencing of HS tetrasaccharides with varying sulfation patterns as well as uronic acid epimerization, including a defined synthetic mixture of 21 tetrasaccharides as well as a mixture of tetrasaccharides from natural HS. Whereas successful separation and identification of nonisomeric HS oligosaccharides was previously achieved, ${ }^{35}$ the current study made it possible to separate and differentiate isomers that are widely found in natural HS (and, by extension, heparin). The synthesis of a series of HS tetrasaccharides with defined structure made it possible to determine the relationship between structures and their chromatographic behavior and fragmentation specificity. The chromatographic selectivity can be affected by three factors including degree of sulfation, sulfation position and uronic 
acid epimerization. While identification of sulfation positional isomers is relatively straightforward after derivatization using diagnostic glycosidic bond cleavage fragments, distinguishing epimers is more challenging. However, through a combination of LC elution time and MS/MS analysis, epimers can be unambiguously differentiated. The initial application of this technology to a simple "heparinome" of naturally occurring HS tetrasaccharides shows the promise that this technology has for high-throughput sequencing of complex mixtures of HS/heparin. For naturally occurring HS tetrasaccharides, assignment of epimerization required the analysis of the sodiated precursor ion, which yielded more complex MS/MS spectra. While the added information on the MS/MS spectra allowed for assignment of the epimerization of uronic acid, the added complexity made manual interpretation much more cumbersome. However, a program for automated annotation of derivatized GAG sequences, which was codeveloped with this project, now allows for the rapid and successful annotation of the more complex MS/MS spectra of the sodiated precursors. ${ }^{53}$ Future applications of the technology can focus solely on the generation and analysis of sodiated precursors, using a combination of automated analysis to assign sites of sulfation and acetylation, with manual interpretation of uronic acid epimerization sites. Applications for sequencing of mixtures of longer HS oligosaccharides from affinity purification or complex biological systems are currently under investigation.

\section{Supplementary Material}

Refer to Web version on PubMed Central for supplementary material.

\section{Acknowledgments}

This research is supported in part by the National Institute of General Medical Sciences-funded "Research Resource for Integrated Glycotechnology" (P41 GM103390) from the National Institutes of Health. The authors gratefully acknowledge Dr. Barry Boyes (Advanced Material Technology, Wilmington, DE) for generously providing us with the custom C18 HALO columns and helpful discussions regarding the reversed phase LC separation.

\section{References}

1. Gandhi NS, Mancera RL. Chem Biol Drug Des. 2008; 72:455-482. [PubMed: 19090915]

2. Rabenstein DL. Nat Prod Rep. 2002; 19:312-331. [PubMed: 12137280]

3. Tumova S, Woods A, Couchman JR. Int J Biochem Cell Biol. 2000; 32:269-288. [PubMed: 10716625]

4. Kresse H, Schonherr E. J Cell Physiol. 2001; 189:266-274. [PubMed: 11748584]

5. Fannon M, Forsten KE, Nugent MA. Biochemistry. 2000; 39:1434-1445. [PubMed: 10684625]

6. Lin X. Development. 2004; 131:6009-6021. [PubMed: 15563523]

7. Wu ZL, Zhang L, Yabe T, Kuberan B, Beeler DL, Love A, Rosenberg RD. J Biol Chem. 2003; 278:17121-17129. [PubMed: 12604602]

8. Perrimon N, Bernfield M. Semin Cell Dev Biol. 2001; 12:65-67. [PubMed: 11292371]

9. Gotte M. FASEB J. 2003; 17:575-591. [PubMed: 12665470]

10. Chen Y, Maguire T, Hileman RE, Fromm JR, Esko JD, Linhardt RJ, Marks RM. Nat Med. 1997; 3:866-871. [PubMed: 9256277]

11. Toyoda H, Kinoshita-Toyoda A, Selleck SB. J Biol Chem. 2000; 275:2269-2275. [PubMed: 10644674]

12. Theocharis AD, Skandalis SS, Tzanakakis GN, Karamanos NK. FEBS J. 2010; 277:3904-3923. [PubMed: 20840587] 
13. Lindahl U, Backstrom G, Hook M, Thunberg L, Fransson LA, Linker A. Proc Natl Acad Sci U S A. 1979; 76:3198-3202. [PubMed: 226960]

14. Guerrini M, Guglieri S, Casu B, Torri G, Mourier P, Boudier C, Viskov C. J Biol Chem. 2008; 283:26662-26675. [PubMed: 18640975]

15. Habuchi H, Habuchi O, Kimata K. Glycoconjugate J. 2004; 21:47-52.

16. Garg HG, Cindhuchao N, Quinn DA, Hales CA, Thanawiroon C, Capila I, Linhardt RJ. Carbohydr Res. 2002; 337:2359-2364. [PubMed: 12433502]

17. Guglier S, Hricovini M, Raman R, Polito L, Torri G, Casu B, Sasisekharan R, Guerrini M. Biochemistry. 2008; 47:13862-13869. [PubMed: 19117094]

18. Nugent MA, Zaia J, Spencer JL. Biochemistry (Moscow). 2013; 78:726-735. [PubMed: 24010836]

19. Zaia J. Mass Spectrom Rev. 2009; 28:254-272. [PubMed: 18956477]

20. Korir AK, Larive CK. Anal Bioanal Chem. 2009; 393:155-169. [PubMed: 18841350]

21. Chi LL, Amster J, Linhardt RJ. Curr Anal Chem. 2005; 1:223-240.

22. Zaia J. Mol Cell Proteomics. 2013; 12:885-892. [PubMed: 23325770]

23. Yang B, Chang Y, Weyers AM, Sterner E, Linhardt RJ. J Chromatogr A. 2012; 1225:91-98. [PubMed: 22236563]

24. Gill VL, Aich U, Rao S, Pohl C, Zaia J. Anal Chem. 2013; 85:1138-1145. [PubMed: 23234263]

25. Maxwell E, Tan Y, Tan Y, Hu H, Benson G, Aizikov K, Conley S, Staples GO, Slysz GW, Smith RD, Zaia J. PLoS One. 2012; 7:e45474. [PubMed: 23049804]

26. Staples GO, Naimy H, Yin H, Kileen K, Kraiczek K, Costello CE, Zaia J. Anal Chem. 2010; 82:516-522. [PubMed: 20000724]

27. Kailemia MJ, Li L, Ly M, Linhardt RJ, Amster IJ. Anal Chem. 2012; 84:5475-5478. [PubMed: 22715938]

28. Leach FE 3rd, Arungundram S, Al-Mafraji K, Venot A, Boons GJ, Amster IJ. Int J Mass Spectrom. $2012 ; 330-332: 152-159$.

29. Wolff JJ, Leach FE 3rd, Laremore TN, Kaplan DA, Easterling ML, Linhardt RJ, Amster IJ. Anal Chem. 2010; 82:3460-3466. [PubMed: 20380445]

30. Huang Y, Yu X, Mao Y, Costello CE, Zaia J, Lin C. Anal Chem. 2013; 85:11979. [PubMed: 24224699]

31. Leach FE 3rd, Ly M, Laremore TN, Wolff JJ, Perlow J, Linhardt RJ, Amster IJ. J Am Soc Mass Spectrom. 2012; 23:1488-1497. [PubMed: 22825742]

32. Wolff JJ, Laremore TN, Aslam H, Linhardt RJ, Amster IJ. J Am Soc Mass Spectrom. 2008; 19:1449-1458. [PubMed: 18657442]

33. Wolff JJ, Laremore TN, Busch AM, Linhardt RJ, Amster IJ. J Am Soc Mass Spectrom. 2008; 19:294-304. [PubMed: 18055211]

34. Oh HB, Leach FE 3rd, Arungundram S, Al-Mafraji K, Venot A, Boons GJ, Amster IJ. J Am Soc Mass Spectrom. 2011; 22:582-590. [PubMed: 21472576]

35. Huang R, Liu J, Sharp JS. Anal Chem. 2013; 85:5787-5795. [PubMed: 23659663]

36. Huang R, Pomin VH, Sharp JS. J Am Soc Mass Spectrom. 2011; 22:1577-1587. [PubMed: 21953261]

37. Lei M, Mechref Y, Novotny MV. J Am Soc Mass Spectrom. 2009; 20:1660-1671. [PubMed: 19546010]

38. Hitchcock AM, Costello CE, Zaia J. Biochemistry. 2006; 45:2350-2361. [PubMed: 16475824]

39. Hitchcock AM, Yates KE, Costello CE, Zaia J. Proteomics. 2008; 8:1384-1397. [PubMed: 18318007]

40. Estrella RP, Whitelock JM, Packer NH, Karlsson NG. Anal Chem. 2007; 79:3597-3606. [PubMed: 17411012]

41. Karlsson NG, Schulz BL, Packer NH, Whitelock JM. J Chromatogr B: Anal Technol Biomed Life Sci. 2005; 824:139-147.

42. Kuberan B, Lech M, Zhang L, Wu ZL, Beeler DL, Rosenberg RD. J Am Chem Soc. 2002; 124:8707-8718. [PubMed: 12121115]

43. Thanawiroon C, Linhardt RJ. J Chromatogr A. 2003; 1014:215-223. [PubMed: 14558627] 
44. Seo Y, Andaya A, Leary JA. Anal Chem. 2012; 84:2416-2423. [PubMed: 22283665]

45. Kailemia MJ, Park M, Kaplan DA, Venot A, Boons GJ, Li L, Linhardt RJ, Amster IJ. J Am Soc Mass Spectrom. 2014; 25:258-268. [PubMed: 24254578]

46. Dell A, Rogers ME, Thomas-Oates JE. Carbohydr Res. 1988; 179:7-19.

47. Arungundram S, Al-Mafraji K, Asong J, Leach FE 3rd, Amster IJ, Venot A, Turnbull JE, Boons GJ. J Am Chem Soc. 2009; 131:17394-17405. [PubMed: 19904943]

48. Pomin VH, Sharp JS, Li X, Wang L, Prestegard JH. Anal Chem. 2010; 82:4078-4088. [PubMed: 20423049]

49. Huang Y, Mao Y, Zong C, Lin C, Boons GJ, Zaia J. Anal Chem. 2015; 87:592-600. [PubMed: 25486437]

50. Ciucanu I, Kerek F. Carbohydr Res. 1984; 131:209-217.

51. Domon B, Costello CE. Glycoconjugate J. 1988; 5:397-409.

52. Rudd TR, Yates EA. Mol BioSyst. 2012; 8:1499-1506. [PubMed: 22370609]

53. Chiu Y, Huang R, Orlando R, Sharp JS. Mol Cell Proteomics. 2015 

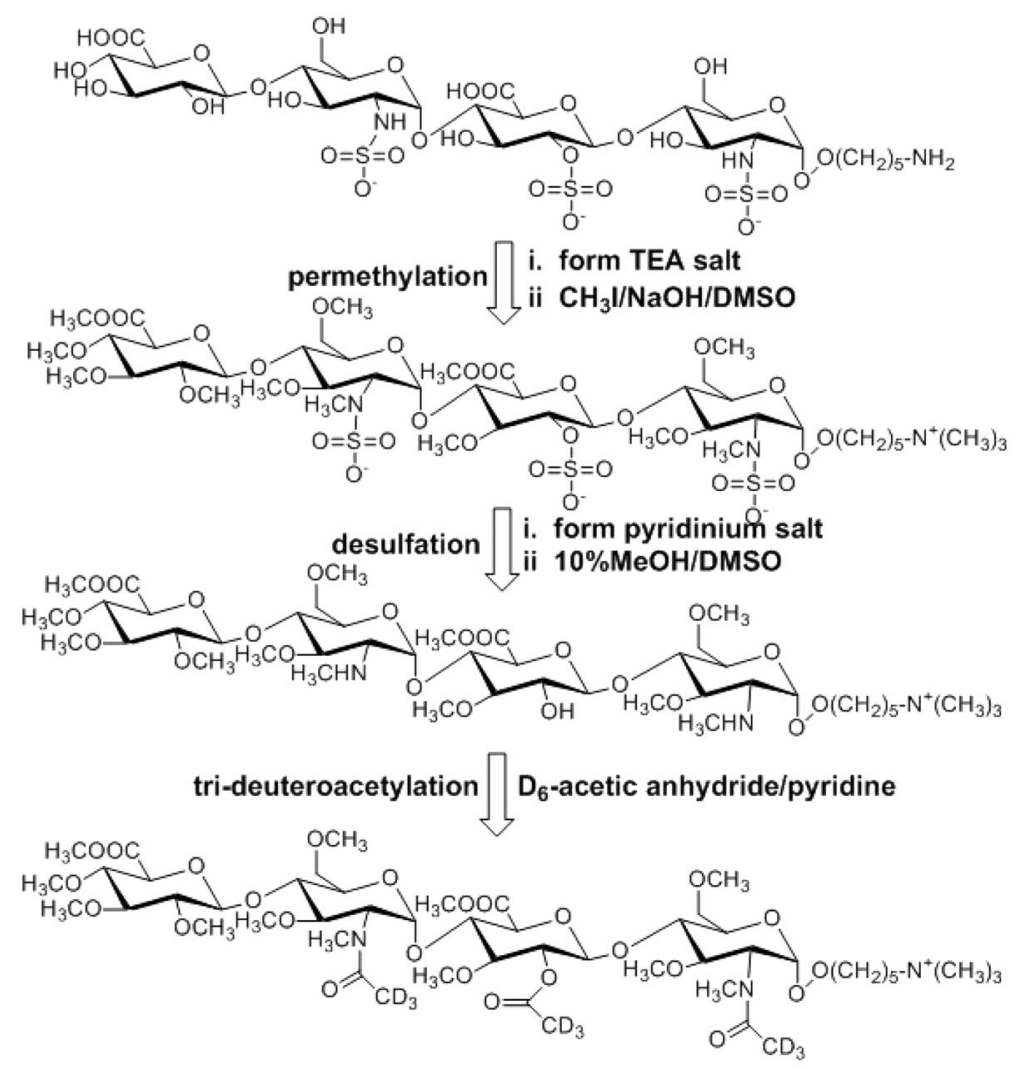

Figure 1.

Structures of the synthetic tetrasaccharide GlcA-GlcNS-GlcA2S-GlcNS6S- $\left(\mathrm{CH}_{2}\right)_{5} \mathrm{NH}_{2}$ (compound 4d) before and after chemical derivatization. Permethylation protects the unsulfated groups, as well as allows the assignment of sulfation sites within the GlcN based on the effects of sulfation on the extent of permethylation. Sulfates are then gently removed by solvolysis, and the sites of sulfation are labeled with trideuteroacetyl groups. The primary amine on the reducing end linker is converted to a quaternary amine during the permethylation, providing a permanent fixed charge on the reducing end. 

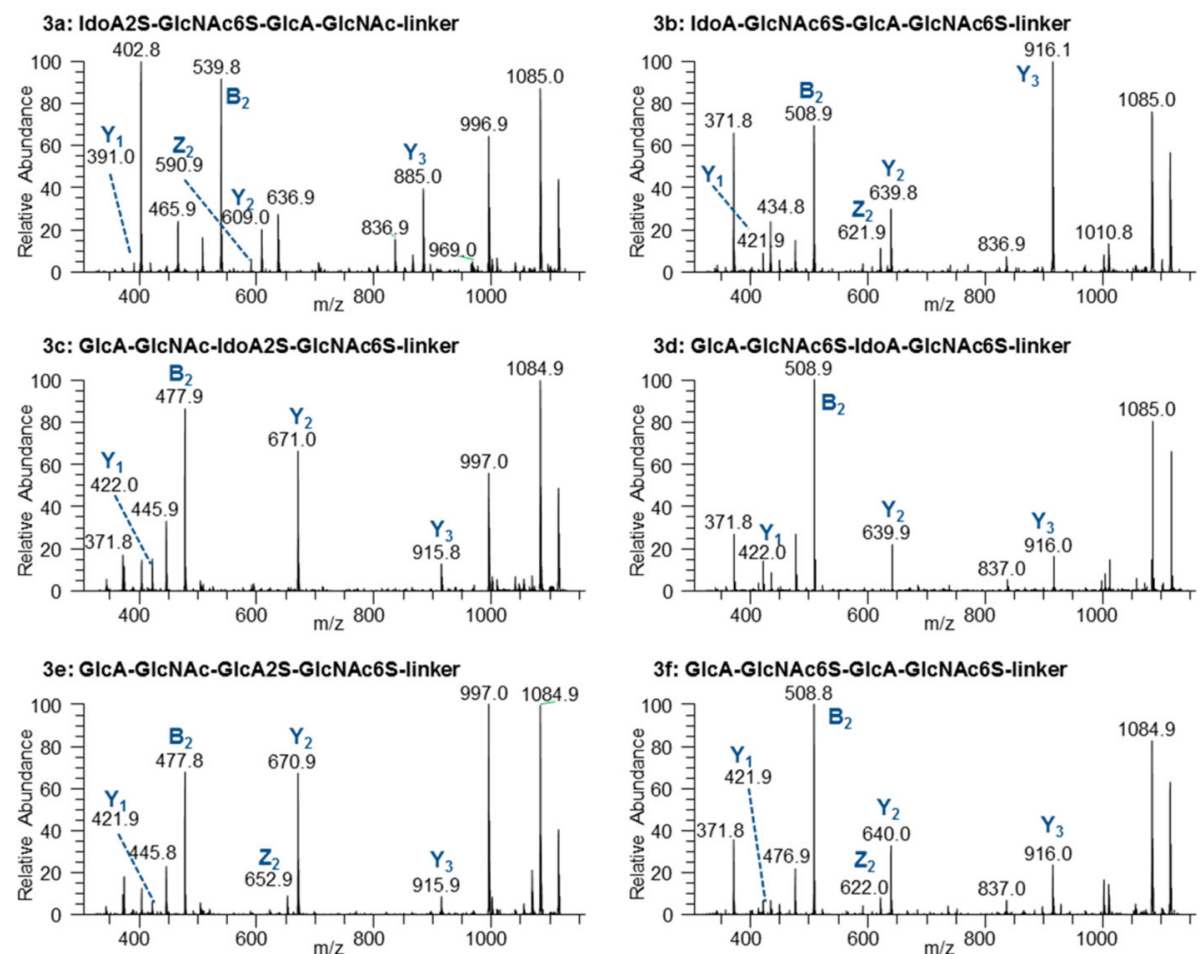

Figure 2.

CID tandem MS spectra for compounds 3a-f with $\mathrm{m} / z$ of 1148.6 for precursor ion $\mathrm{M}^{+}$. These six tetrasaccharides could be divided into three isomeric sets: [3a], [3b, 3d, 3f], and [3c, 3e], where tetrasaccharides within each set were epimers. Sequential Y ions for each tetrasaccharide were observed that could be used as diagnostic ions to identify sulfation positional isomers. Differences of the relative intensities ratio of certain fragment ions were also observed within epimers. For example, compounds 3c-f containing terminal GlcA had $\mathrm{Y}_{3} / \mathrm{Y}_{2}$ ratio $<0.8$, whereas compounds $\mathbf{3 a}$ and $\mathbf{3 b}$ containing terminal IdoA shown $\mathrm{Y}_{3} / \mathrm{Y}_{2}$ ratio $>0.8$. On the other hand, $\mathrm{Z}_{2}$ ions were observed for compounds $\mathbf{3 a - f}$ containing internal GlcA residue, but not for compounds $\mathbf{3 c}$ and $\mathbf{3 d}$ containing internal IdoA instead. 

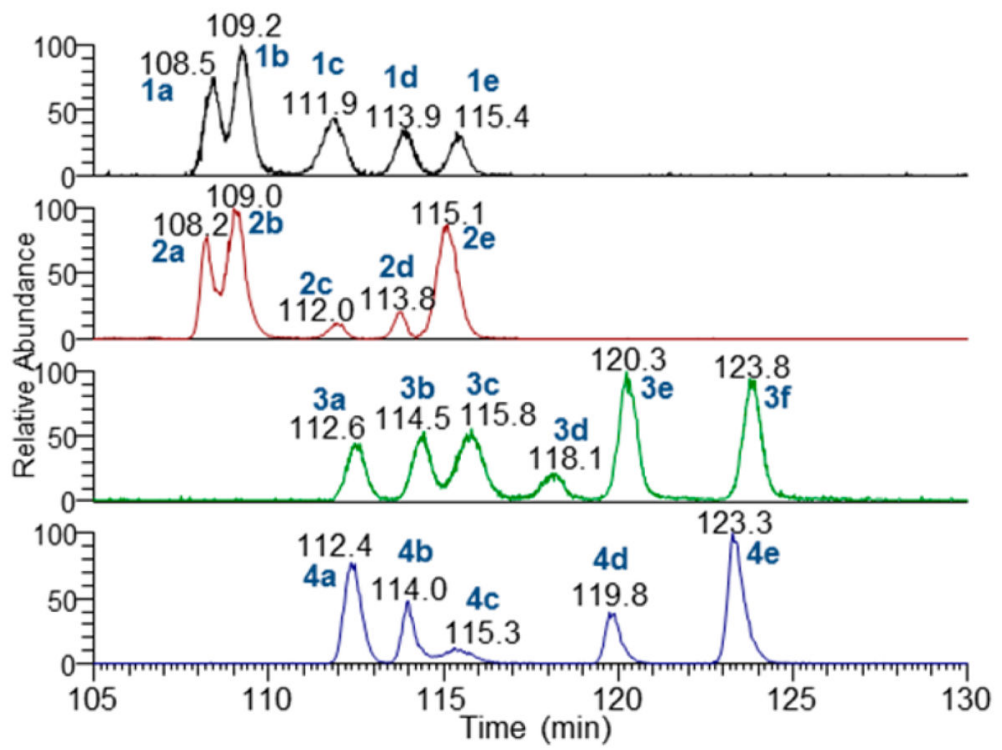

Figure 3.

Reversed phased LC separation for derivatized synthetic tetrasaccharide mixture with alkyl linkers. Extracted ion charomatograms (EIC) for each of the four precursor ions were presented, with a total of 21 tetrasaccharides being separated. Peaks are labeled with the compound \# of the eluting tetrasaccharide. Refer to Table 1 for tetrasaccharides structures. 


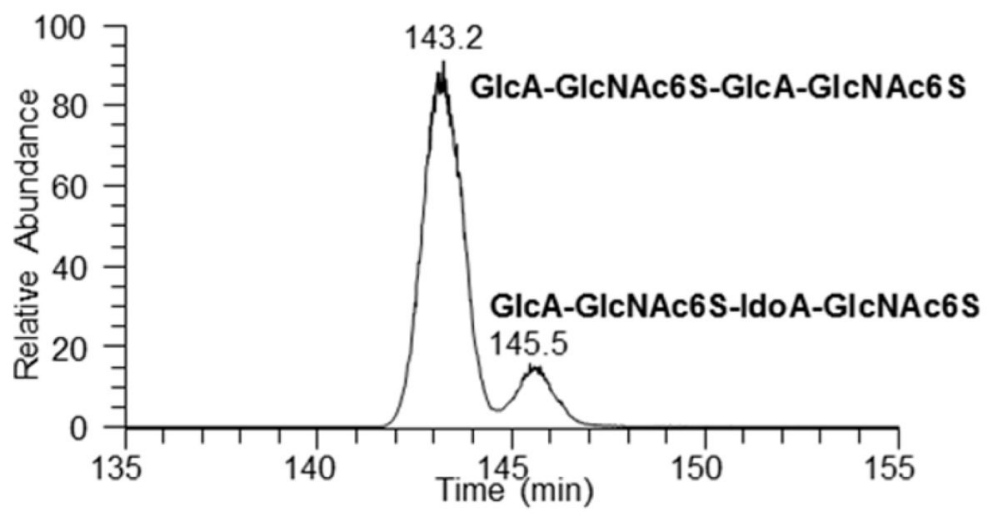

GlcA-GlcNAc6S-GlcA-GlcNAc6S

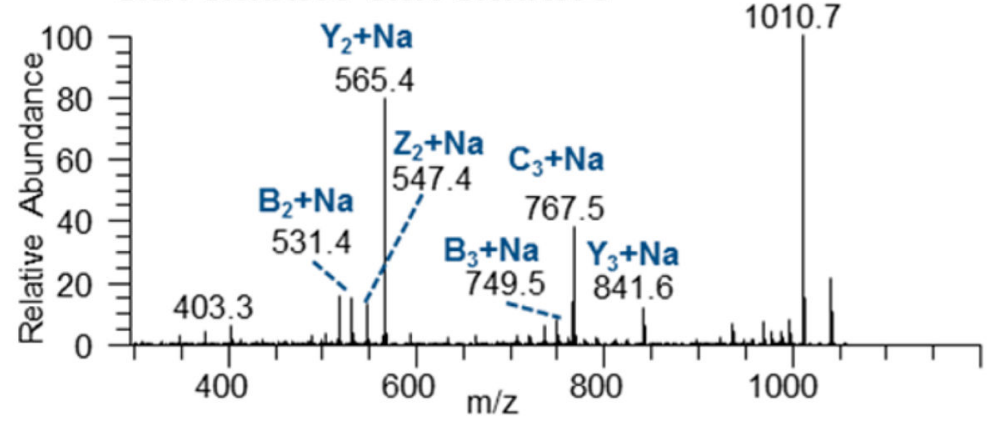

GIcA-GIcNAc6S-IdoA-GIcNAc6S

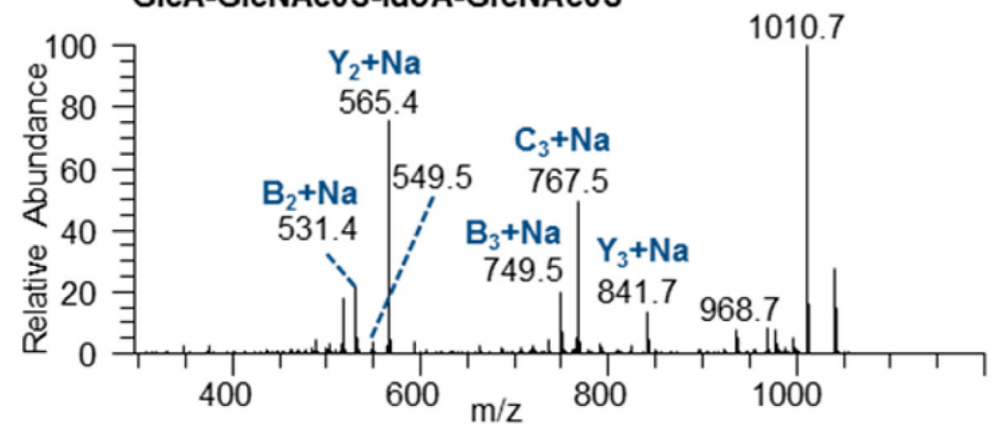

Figure 4.

LC chromatogram (A) and MS/MS spectra of the tetrasaccharide standards GlcAGlcNAc6S-GlcA-GlcNAc6S (B) and GlcA-GlcNAc6S-IdoA-GlcNAc6S (C) of parent ion $[\mathrm{M}+\mathrm{Na}]^{+}$. A series of $\mathrm{B}$ ions and $\mathrm{Y}$ ions were observed for sequencing of sulfation positions, whereas $\mathrm{Z}_{2}$ ion with $\mathrm{m} / \mathrm{z}$ of 547.4 was only observed for GlcA-GlcNAc6S-GlcAGlcNAc6S with internal GlcA residue. 


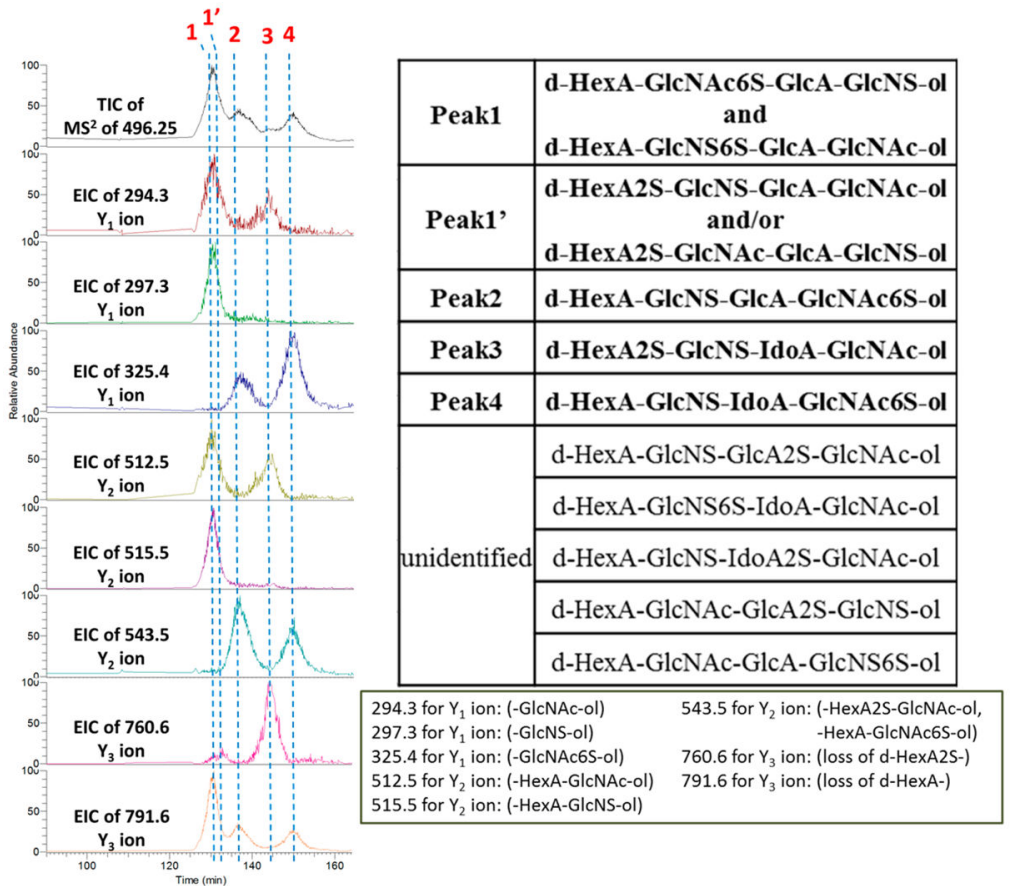

Figure 5.

LC separation and sequencing of one postderivatized composition (with MW of 990. 490) of the native HS tetrasaccharide mixture. The TIC of MS/MS of parent ion $[\mathrm{M}+2 \mathrm{H}]^{2+}(\mathrm{m} / z$ of 496.25) and a series of EICs of diagnostic Y ions (left). EIC were extracted using the indicated $\mathrm{m} / \mathrm{z}$ value with a window of $1 \mathrm{Da}$. Three major peaks $(1,2$, and 4$)$ and two minor peaks ( 3 and $1^{\prime}$ ) were observed. A list of total 12 biosynthetically possible theoretical structures for this specific MW is shown (top right), with identified structures shown in bold. The structural assignment(s) of each fragment ion mass is also shown (bottom right). 


\section{Table 1}

Sequences for Four Sets of Synthetic Tetrasaccharides with Alkyl Linker at the Reducing End ${ }^{a}$

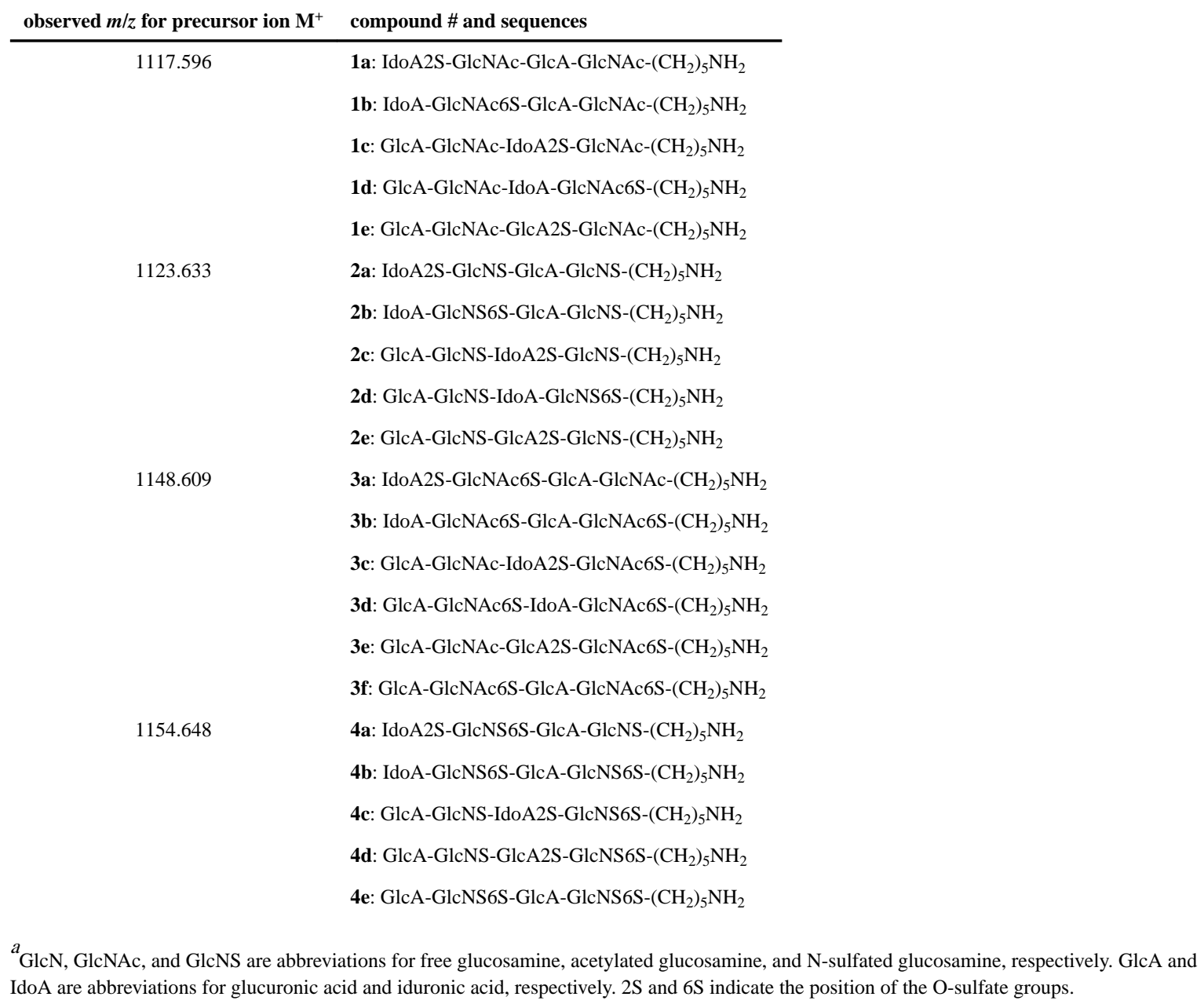

${ }^{a}$ GlcN, GlcNAc, and GlcNS are abbreviations for free glucosamine, acetylated glucosamine, and N-sulfated glucosamine, respectively. GlcA and IdoA are abbreviations for glucuronic acid and iduronic acid, respectively. $2 \mathrm{~S}$ and $6 \mathrm{~S}$ indicate the position of the O-sulfate groups. 